\title{
POD Analysis of Cavity Flow Instability
}

\author{
A. Rona* and E.J. Brooksbank ${ }^{\dagger}$ \\ Department of Engineering, \\ University of Leicester LE1 7RH, UK
}

\begin{abstract}
A Mach 1.5 turbulent cavity flow develops largeamplitude oscillations, pressure drag and noise. This type of flow instability affects practical engineering applications, such as aircraft store bays. A simple model of the flow instability is sought towards developing a real-time model-based active control system for simple geometries, representative of open aircraft store bays.

An explicit time marching second-order accurate finite-volume scheme has been used to generate timedependent benchmark cavity flow data. Then, a simpler and leaner numerical predictor for the unsteady cavity pressure was developed, based on a Proper Orthogonal Decomposition of the benchmark data.

The low order predictor gives pressure oscillations in good agreement with the benchmark CFD method. This result highlights the importance of large-scale phase-coherent structures in the Mach 1.5 turbulent cavity flow. At the selected test conditions, the significant pressure 'energy' content of these structures enabled an effective reduced order model of the cavity dynamic system. Directions and methods to further streamline and simplify the unsteady pressure predictor have been highlighted.
\end{abstract}

\section{Introduction}

The unsteady flow in an enclosure, or cavity, open to a turbulent transonic air stream is characterised by large amplitude pressure oscillations, an unsteady vorticity field and flow recirculation (Fig. 1). This leads to sustained aerodynamic loading on the solid walls, pressure drag and noise. Cavity flows develop between train sections, in automotive components and in aircraft landing wells. In the transonic flow regime, aircraft store bays have been a very prominent application in recent years. ${ }^{1}$ In fact, current airframe designs make extensive use of internal stores for stealth and store protection during cruise. When the store bay is opened during flight, the stores may be subjected to pressure fluctuations in excess of $150 \mathrm{~dB}$ re $20 \mu \mathrm{Pa}$ and this level of structural excitation could undermine the integrity of a store. ${ }^{2}$ Safe store separation is also a concern, as light stores may impact the aircraft during store ejection, due to the unsteady flow.

\footnotetext{
* Lecturer

${ }^{\dagger}$ Research Associate.
}

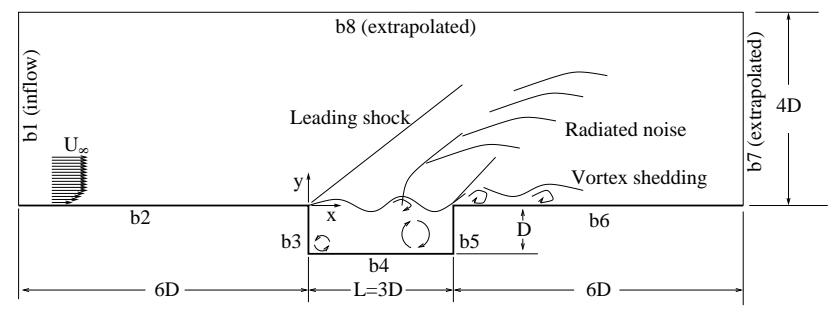

Fig. 1 Sketch of the supersonic flow past an open cavity.

Past experiments ${ }^{3-5}$ and numerical studies ${ }^{1,6}$ have shown that an attenuated flow instability can be achieved with ramps, ${ }^{7-11}$ spoilers, rods $^{12}$ or air jets ${ }^{4,13,14}$ acting on the oncoming flow. When active control devices are used, such as vibrating ramps ${ }^{2,15}$ or pulsating jets, ${ }^{4,14,16}$ the cavity flow dynamics interacts with the actuator flow. At subsonic speeds, linear models of the flow dynamics give a powerful description of such interaction and highlight the performance limitation boundaries of linear control schemes. ${ }^{17}$

This paper aims to characterise the dynamic system of a supersonic turbulent cavity flow. At this regime, limit-cycle self-sustained instabilities may be encountered ${ }^{18-20}$ that require a non-linear modelling approach. Specifically, a Proper Orthogonal Decomposition (POD) of the flow ${ }^{21-23}$ is used to qualify the dynamic system in the enclosure. Then, extending this modal analysis approach, the objective is to derive a simplified dynamic model. This model should retain a good fidelity to the real or benchmark model flow, it should be computationally lean and relatively simple to implement and should give an additional insight into the dynamics of the supersonic flow regime.

A Mach 1.5 flow over a rectangular enclosure of length to depth ratio 3 forms an 'open' cavity. ${ }^{24}$ The flow instability is driven by the fluctuations of a shear layer that spans across the enclosure. ${ }^{24}$ The interaction of the shear layer with the rear bulkhead is part of a feed-back loop which self-sustains the instability. This configuration was studied experimentally at Cambridge ${ }^{25}$ and then further numerical work was conducted at the University of Southampton, ${ }^{19,20,26}$ leading to benchmark data to develop the reduced order dynamic model that is presented in this paper. Other flow regimes and geometries have been investigated independently and a useful collection of such work is given by Grace. ${ }^{6}$

1 of 9

American Institute of Aeronautics and Astronautics 
The Mach 1.5 cavity flow geometry considered in this study features large-scale convecting instabilities that characterise the unsteady shear layer over the cavity opening. These instabilities are the energy containing eddies in the shear layer and were found to be the main drivers of the self-sustained flow unsteadiness. ${ }^{19,20,26}$ The use of active controllers to suppress the cavity flow instability and minimise pressure fluctuations requires the implementation of control devices. In order to design practicable, implementable real-time feedback controllers, it is necessary to have a control device that is computationally efficient. Such a controller requires a flow predictor as input to the system.

The Proper Orthogonal Decomposition (POD) method, ${ }^{27}$ also known as principal component analysis or Karhunen-Loève expansion, is a technique used to capture the overall behaviour of a dynamic system and is used in the current study as a flow predictor. The method generates an eigenvector matrix that captures the non-linearity of the input system with the advantages that the eigenvector elements are ordered from the first element with the highest average energy to the element with the lowest average energy. This is an optimal basis from which to reconstruct the unsteady flow, as the first elements contain most of the energy of the flow. In this approach, a desired energy level can be specified a priori and only those eigenvectors or basis elements needed to achieve such a level are stored to project the flow prediction in time.

In this work, a further development is introduced with respect to past applications of the POD technique to the transonic cavity flow. The POD analysis delivers what is essentially a low order description of the flow, based on a spatial decomposition in a small number of modes. The dynamic cavity problem is therefore simplified by the POD analysis in wavenumber space. A discrete Fourier transform is added in this work to obtain a reduced dynamic cavity model in the wavenumber and frequency domains. This simplification in frequency derives from the dominance of large scale structures and of their associated convective or characteristic velocities in the unsteady flow. At certain flow conditions, the flow structures and their characteristic velocities may become locked in a self-sustained instability, giving good phase coherence between successive oscillations. This should enable the extrapolation in time, over a few characteristic periods, of the contribution from such phase-coherent modes to the overall flow unsteadiness. Such extrapolation may capture enough kinetic energy of the cavity flow to lead to the design of an active flow controller, based on the predictions from this reduced order model of the unsteady flow.

\section{Flow Geometry}

In this study, a rectangular enclosure of length to depth ratio 3 is considered, as shown in Fig. 1. The rectangular cutout is located at the centre of a flat plate, equispaced between the inflow and the outflow boundaries. From the inflow boundary, the upstream edge of the plate leading to the enclosure is twice the cavity in length, so is the plate from the rear end of the enclosure to the outflow boundary. Past experiments $^{25}$ and numerical studies ${ }^{19}$ have shown that, at high Reynolds numbers, a cavity flow unsteadiness develops within the enclosure and its immediate surroundings. By defining the inflow and outflow boundaries as shown in Fig. 1, the most active flow regions are covered by the computational domain. ${ }^{28}$

At the inflow, a turbulent boundary layer velocity profile is imposed. The boundary layer thickness $\delta_{99}$ is $0.333 D$, where $D=15 \mathrm{~mm}$ is the cavity depth. Above the boundary layer, the inflow is uniform and supersonic. The free stream Mach number $M_{\infty}$ is 1.5 , the free stream velocity $U_{\infty}=425.2 \mathrm{~m} / \mathrm{s}$ is parallel to the plate, the free stream density $\rho_{\infty}$ is $0.9373 \mathrm{~kg} / \mathrm{m}^{3}$, the static pressure $p_{\infty}=53.801 \mathrm{kN} / \mathrm{m}^{2}$ and the static temperature $T_{\infty}=200 \mathrm{~K}$. These flow conditions are selected to match an experimental test case documented in Zhang. ${ }^{25}$ All cavity flow results are normalised by the above free stream values and $D / U_{\infty}$ normalises time.

At these inlet conditions, the cavity flow is 'open'24 and the instability is shear layer driven as opposed to a wake mode. ${ }^{29,30}$ The supersonic inflow limits the upstream propagation of disturbances from the enclosure to a narrow flow region below the sonic line in the wall boundary layer that develops over the cavity leading edge. This helps to reduce any wave reflection from the upstream computational boundary. ${ }^{28}$ Nonslip conditions are imposed on the solid walls by the use of a wall function. Spalding's law of the wall is used to estimate the tangential velocity. The law of the wall is implemented with a von Karman constant $\kappa=0.41$ and $B=5.0 .^{14}$

\section{Numerical Method}

A turbulent cavity flow model is obtained by discretising the short-time averaged Navier-Stokes equations $^{31}$ that are the governing equations for the compressible cavity flow. Turbulence closure is obtained with a $k-\omega$ turbulence model that includes crossdiffusion of specific turbulent dissipation rate. ${ }^{32}$

A rectangular regular mesh of $200 \times 160$ unit control volumes or cells forms the computational domain outside the cavity and a $40 \times 40$ mesh is used inside the enclosure. A unit control volume aspect ratio of three is used throughout the domain. The flow governing equations are integrated at the unit cell boundaries

2 of 9

American Institute of Aeronautics and Astronautics 


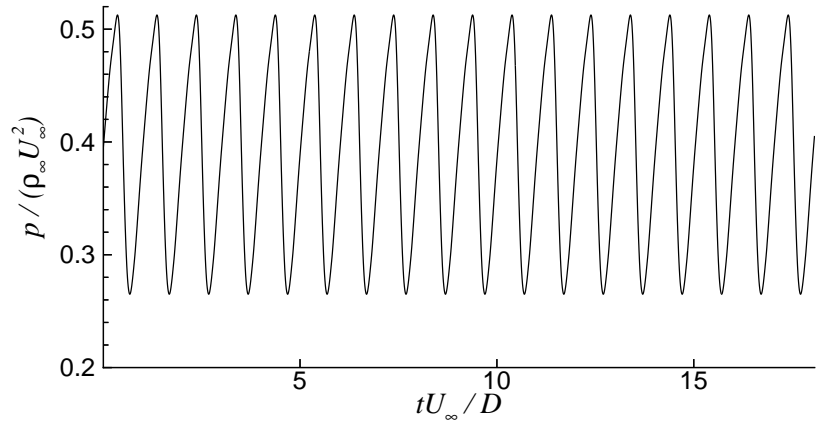

Fig. 2 Normalised pressure fluctuations at the downstream cavity edge over 18 periods (18T).

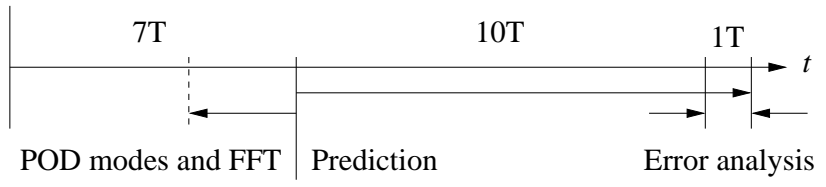

Fig. 3 Subdivision of the 18 period timeline.

by a finite volume scheme. In this scheme, the convective fluxes are estimated using a second order accurate flux-limited numerical method with the min-mod flux limiter of Roe. ${ }^{33,34}$ The diffusive fluxes are estimated by second order central differencing. The numerical simulation is time-marched using the $\mathrm{Hu}$ et al. ${ }^{35}$ low storage implementation of an explicit two-step RungeKutta integration scheme. This scheme uses standard Runge-Kutta coefficients 0.5 and 1.0 to maintain second order formal accuracy in time. A constant time step of $\Delta t=0.00435 D / U_{\infty}$ is used throughout the computation, which corresponds to a Courant number of approximately 0.13 . Further details of the numerical method are reported in previous work. ${ }^{14,28,36}$

After priming the flow in the computational domain, ${ }^{28}$ the flow history is advanced until a selfsustained oscillatory flow regime is reached. ${ }^{14}$ The wall pressure at the downstream cavity edge is monitored during this initial phase of the computation. The computation is deemed to have reached a selfsustained flow regime when the monitored pressure becomes statistically stationary. Figure 2 shows the normalised wall pressure at the downstream edge over a time span of approximately $18 D / U_{\infty}$, following this initial settling time. The oscillations appear selfsimilar and statistically invariant over time. In Fig. 2, 18 pressure maxima identify 18 periods $T$ of instability. As shown in Fig. 3, the first 7 periods are used for a POD analysis of the flow and the 18 th period is used to compare the CFD results against those from the fast flow predictor described in the following section.

\section{Fast Flow Predictor}

\section{Method overview}

From the flow state in the enclosure at any given time $t_{0}$, a method is sought to predict the flow at a later time $t>t_{0}$. The current time accurate computational fluid dynamic method is one way of obtaining such prediction everywhere in the computational domain. In spite of the on-going advances in the CPU performance of computer hardware, this method cannot yet deliver predictions in real-time that may be used by a real-time control algorithm to actively suppress the flow instability. A simpler predictive tool is therefore sought with the potential to deliver realtime predictions. One simplification available to build such tool is that, as flow control aims to reduce the unsteady pressure on the cavity walls, an extrapolation in time of the pressure field alone can be sought. This reduces the computational task with respect to predicting all the conservative variables in the flow. A second simplification, not yet applied to this study, is that time dependent pressure predictions are required only along solid boundaries, where the potential for structural damage in an empty cavity configuration is of concern. This second aspect may lead to further streamlining of the predictive tool in future work.

To extrapolate the cavity pressure field in time, a two-stage approach is followed. The first stage consists of performing a Proper Orthogonal Decomposition (POD) analysis on the known flow field at time $t_{0}$ and on its past pressure history, in the range $0 \leq t<t_{0}$. The second stage uses an inverse discrete Fourier series of the POD modes from the first stage to estimate the unknown unsteady pressure field at a later time $t>t_{0}$.

\section{Part 1: Proper Orthogonal Decomposition}

The first stage of the pressure prediction method is to analyse a known pressure history by a Proper Orthogonal Decomposition. The aim is to construct a set of time-independent POD bases that maximise the $L_{2}$ norm of the pressure field. Let $\boldsymbol{X}=\left\{\boldsymbol{x}_{1}, \boldsymbol{x}_{2}, \ldots, \boldsymbol{x}_{N}\right\}$ be a matrix of $N$ snapshots of the flow field, in which $\boldsymbol{x}_{i}$ is a concatenation of the two-dimensional pressure field into a column vector of length $M$. A mean pressure field is constructed from the snapshots:

$$
\overline{\boldsymbol{x}}=\frac{1}{N} \sum_{i=1}^{N} \boldsymbol{x}_{i}
$$

to give a zero mean pressure data $\tilde{\boldsymbol{X}}=\left\{\left(\boldsymbol{x}_{1}-\overline{\boldsymbol{x}}\right),\left(\boldsymbol{x}_{2}-\right.\right.$ $\left.\overline{\boldsymbol{x}}), \ldots,\left(\boldsymbol{x}_{N}-\overline{\boldsymbol{x}}\right)\right\}$. A correlation matrix $\boldsymbol{C}$ is formed from the zero mean pressure data:

$$
\boldsymbol{C}=\tilde{\boldsymbol{X}}^{T} \tilde{\boldsymbol{X}}
$$

A singular value decomposition rearranges $\boldsymbol{C}$ in the following matrix product:

$$
\boldsymbol{C}=\boldsymbol{V} \boldsymbol{\Lambda} \boldsymbol{V}^{T}
$$

where the trace of the diagonal matrix $\boldsymbol{\Lambda}$ is populated by the eigenvalues $\lambda_{1} \geq \lambda_{2} \geq \ldots \geq \lambda_{N}$ of $\boldsymbol{C}$ and $\boldsymbol{V}$ is the matrix of the associated eigenvectors. 
A proper orthogonal decomposition basis $\boldsymbol{\Phi}=$ $\left\{\phi_{1}, \phi_{2}, \ldots, \phi_{N}\right\}$ is obtained from

$$
\Phi=\tilde{X} \boldsymbol{V}
$$

A coefficient matrix is constructed from

$$
\boldsymbol{A}=\boldsymbol{C} \boldsymbol{V}=\left(\alpha_{i k}\right)
$$

where subscript $k$ denotes the $k$ th POD mode and subscript $i$ denotes the $i$ th snapshot coefficient of POD mode $k$. Each snapshot $i$ can then be reconstructed to a pre-defined degree of accuracy by

$$
\hat{\boldsymbol{x}}_{i}=\overline{\boldsymbol{x}}+\sum_{k=1}^{P} \alpha_{i k} \boldsymbol{\phi}_{k}
$$

If $P=N$ and $N$ is the number of original snapshots, the snapshots are reconstructed exactly. An approximation can be achieved, to an accuracy of $Q \%$, by taking the first $P<N$ eigenvectors such that ${ }^{21}$

$$
\sum_{k=1}^{P} \lambda_{k} / \sum_{k=1}^{N} \lambda_{k}>\frac{Q}{100}
$$

\section{Part 2: Discrete Fourier Series}

At certain flow conditions, self-sustained cavity flow oscillations are limit-cycle instabilities, featuring dominant modes at specific frequencies and wavenumbers. ${ }^{22}$ This leads to a kinetic energy spectrum characterised by narrow-band frequency peaks dominating over a broad-band floor of background kinetic energy. Most of the kinetic energy of the unsteady flow is concentrated around a few dominant modes in a limitcycle cavity oscillation. In Eq. 5, the time-dependent coefficients $\alpha_{i k}$ represent the squared pressure amplitude of specific POD modes and they are likely to be characterised by a similar dominant narrow-band frequency content. Under this assumption, these coefficients can be approximated by a truncated discrete Fourier series, leading to an approximate set of coefficients

$$
\hat{\alpha}_{i k}=\chi_{k}+\sum_{l=1}^{R}\left[\beta_{k, l} \cos \left(\omega_{k, l} i \Delta t\right)+\delta_{k, l} \sin \left(\omega_{k, l} i \Delta t\right)\right]
$$

where $k$ denotes the number of the POD basis vector. $l$ is the $l$ th Fourier coefficient of mode $k$ and $R$ is the total number of Fourier modes in the truncated series. In this study, the series has been truncated to $R=$ 6. Provided the flow instability is reasonably phasecoherent, Eq. 8 provides an estimate of $\alpha_{i k}$ beyond the known flow history $0 \leq t \leq t_{0}=N \Delta t$. This is obtained by simply substituting $i>N$ in Eq. 8 to evaluate $\hat{\alpha}_{i k}$ at $t>N \Delta t$. By analogy with Eq. 6, a snapshot prediction of the flow at $t>N \Delta t$ is given by

$$
\hat{\boldsymbol{x}}_{i}=\overline{\boldsymbol{x}}+\sum_{k=1}^{P} \hat{\alpha}_{i k} \boldsymbol{\phi}_{k}
$$

Equation 9 therefore allows to extrapolate the flow history beyond the initial data set without having to time march through intermediate time steps, unlike in the finite volume CFD method. This is a key feature to speed-up the flow prediction with respect to a direct CFD time-marching approach.

In Eq. 8, the time-independent coefficient $\chi_{k}$, the Fourier pair $\beta_{k, l}$ and $\delta_{k, l}$ and the mode angular frequencies $\omega_{k, l}(\mathrm{rad} / \mathrm{s})$ are determined from the POD results for $\alpha_{i k}$ from the known flow history $0 \leq t \leq N \Delta t$. $\chi_{k}$ is a discrete time average of $\alpha_{i k}$ and is obtained from

$$
\chi_{k}=\frac{1}{N} \sum_{i=1}^{N} \alpha_{i k}
$$

Then, the leading terms $\left(\omega_{k, 1}, \beta_{k, 1}\right.$ and $\left.\delta_{k, 1}\right)$ of the remaining unknowns $\left(\omega_{k, l}, \beta_{k, l}\right.$, and $\left.\delta_{k, l}\right)$ are evaluated from a discrete Fourier transform on $\alpha_{i k}, i \leq N$. This gives the narrow-band discrete power spectral density of $\alpha_{i k}$. The centre-band frequency of the highest power spectral density peak identifies $\omega_{k, 1}$, while the narrowband SPL and phase at $\omega_{k, 1}$ gives a first estimate of the Fourier pair $\beta_{k, 1}$ and $\delta_{k, 1}$. These estimates are then refined by minimising the cumulative squared error

$$
e_{k}^{2}=\sum_{i=1}^{N}\left(\alpha_{i k}-\hat{\alpha}_{i k}\right)^{2}
$$

using a Newton-Raphson iteration. To determine $\beta_{k, l}$, $\delta_{k, l}$ and $\omega_{k, l}$ for $l>1$, the residual POD mode energy is estimated from

$e_{i k}=\alpha_{i k}-\chi_{k}-\sum_{l=1}^{R}\left[\beta_{k, l} \cos \left(\omega_{k, l} i \Delta t\right)+\delta_{k, l} \sin \left(\omega_{k, l} i \Delta t\right)\right]$

with $R=1$. A discrete Fourier transform is performed on $e_{i k}, i \leq N$, to obtain the second spectral peak frequency $\omega_{k, 2}$. Then $\beta_{k, 2}$ and $\delta_{k, 2}$ are determined following the same Newton-Raphson optimisation as for $\beta_{k, 1}$ and $\delta_{k, 1}$. Higher Fourier pair numbers $(l>2)$ are obtained in the same way.

\section{Results}

\section{Baseline cavity flow}

The numerical model predicts an unsteady flow inside and around the enclosure. This unsteadiness is evident from the wall pressure traces in Fig. 4, obtained at four monitoring positions around the enclosure perimeter. These positions are specified in the legend of Fig. 4 with respect to the Cartesian frame of reference shown in Fig. 1. The pressure fluctuations appear to be periodic and self-similar over time. The longer time pressure history in Fig. 2 confirms this periodicity. This is characteristic of a saturated flow instability in which a limit-cycle behaviour is reached at the system eigen-frequencies. As in experiment, ${ }^{25}$

4 of 9

American Institute of Aeronautics and Astronautics 


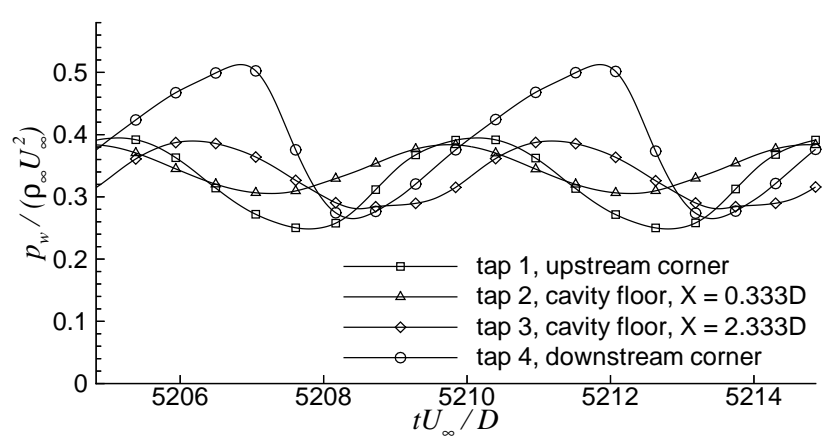

Fig. 4 Baseline cavity surface pressure histories over two dominant eigen-mode periods $(2 T)$.

the dominant mode is the second Rossiter mode and two periods of this mode are shown in Fig. 4. The pressure fluctuation is highest in peak to peak amplitude at the downstream cavity edge $(x=L, y=0 D)$ and is significant over the cavity floor and at the upstream edge.

Figure 5 shows four snapshots of the predicted cavity pressure field, spanning one period $T$ of the leading instability mode, as identified from Fig. 2. The snapshots are evenly spaced in time at a phase lag of $\pi / 2$ to each other. Along the cavity open side, pressure minima mark the position of convecting vortex cores moving in the downstream direction. These vortices roll-up in the separating boundary layer at approximately $0.5 D$ from the upstream edge. The pressure minimum corresponding to the centre of a newly rolled-up vortex is shown in Fig. 5(d) at about $(x=1 D, y=0 D)$ along the shear layer. In Fig. 5(a), this vortex core has moved parallel to the free stream to $(2 D, 0 D)$ and is shown approaching the cavity trailing edge in Fig. 5(b). Upon reaching the rear end, the convecting vortex is sheared and stretched as it interacts with the solid boundary, generating a pressure fluctuation according to the compressible form of the Poisson momentum equation. In Fig. 5(c), the pressure rise due to this vortex stretching of $\nabla \cdot(\boldsymbol{u} \times \boldsymbol{\omega})$, where $\boldsymbol{\omega}=\nabla \times \boldsymbol{u}$, is shown inside the cavity as a packing of the contours, normal to the flow, in the range $2 D \leq x \leq 2.5 D$. Outside the enclosure, these pressure contours bend clockwise towards the downstream direction and stand at a Mach angle $\left(=\arcsin M^{-1}\right)$ corresponding to $M=2.5$ to the free stream direction. This indicates that the contours identify a pressure wave that is moving upstream at about the speed of sound inside and outside the cavity. In Fig. 5(d), this upstream travelling pressure wave has reached $x \sim 0.7 D$ inside the enclosure. Upon reaching the upstream cavity wall in Fig. 5(a), the portion of the wave inside the enclosure is reflected back, while the wave branch above $y=0$ runs off along the unsteady leading edge shock to the far-field, where it would be perceived as aerodynamic noise.
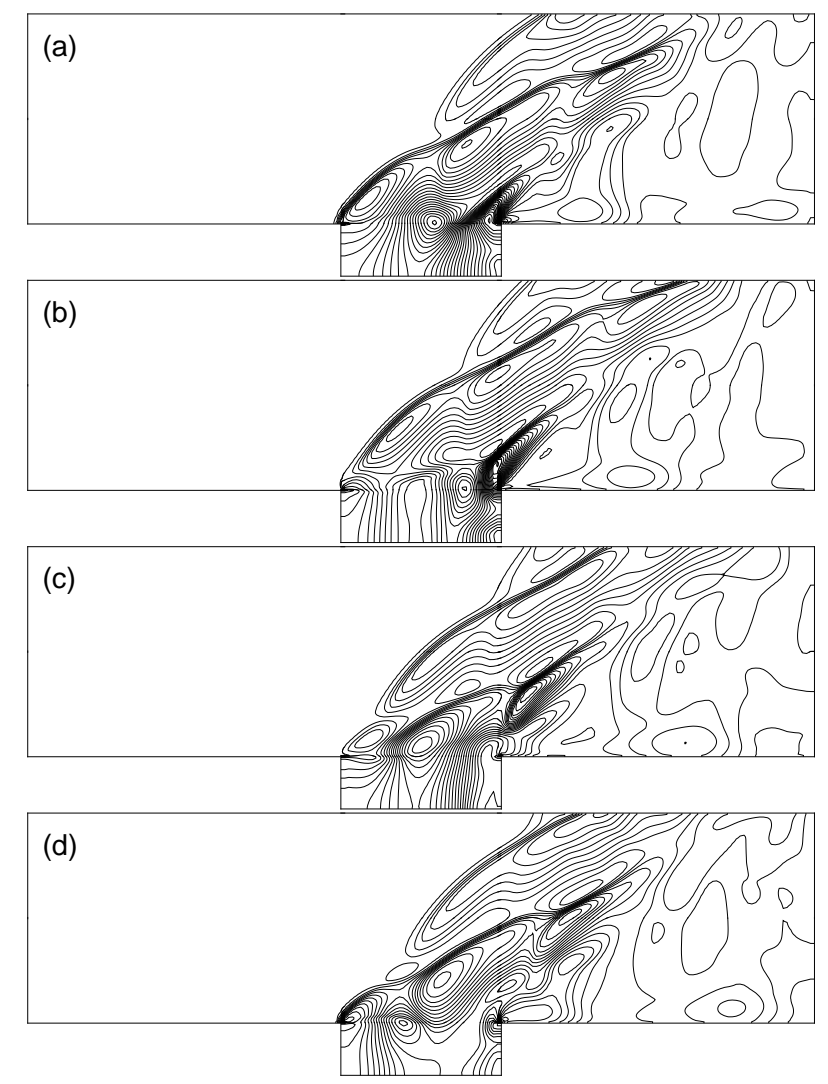

Fig. 5 Baseline cavity pressure contours over one dominant eigen-mode period $T$. (a) $t=0$, (b) $t=$ $0.25 T$, (c) $t=0.5 T$, (d) $T=0.75 T . \Delta p=0.003 \rho_{\infty} U_{\infty}^{2}$.

The bending of the pressure contours from being normal to the flow to standing at about an arcsin $2.5^{-1}$ Mach angle to the free stream in Fig. 5(c) identifies the presence of a shear layer across the cavity opening. This shear layer spans the opening from the upstream to the downstream edge and reattaches on the downstream wall. This 'open' cavity flow regime ${ }^{24}$ was also observed in the experiment through spark schlieren flow visualisation. ${ }^{26}$ The downstream convecting vortex cores are synchronous with the upstream propagation of pressure waves. These two processes form part of a feed-back loop that self-sustains the open cavity flow instability. ${ }^{37}$ In addition, the unsteady flow recirculation inside the cavity is known to aid the feed-back process at certain inflow conditions. ${ }^{38}$

\section{POD modes of the cavity flow}

A proper orthogonal decomposition has been performed on the cavity flow history predicted by the time accurate CFD method. 406 snapshots of the pressure field, similar to those of Fig. 5(a-d), have been used. These snapshots cover approximately 2.0 periods of the dominant second Rossiter ${ }^{37}$ cavity mode. The POD method orders the modes from the most energetic to the least energetic. In Fig. 6 the cumulative sum of the $L_{2}$ norm of the first 16 modes is 


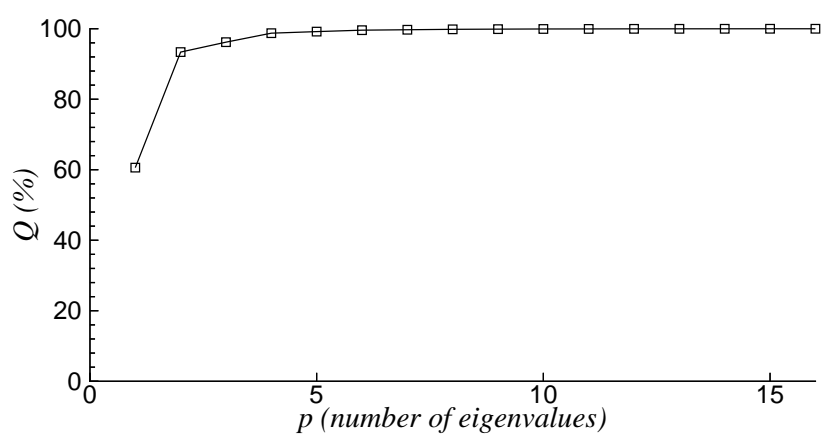

Fig. 6 Cumulative energy distribution of the first 16 most energetic modes.

shown, normalised with respect to the mean squared pressure. Adding the first 9 modes gives $99.9 \%$ of the mean squared pressure in the 406 snapshots cavity flow history. This corresponds to $Q=99.9 \%$ in Eq. 7. This analysis clearly indicates that most of the flow 'energy' is concentrated in few spatial modes and that a modal approach to the prediction of cavity flow captures the key features of the modelled self-sustained instability.

The mean pressure field and the first 4 out of the 16 POD modes sequence is shown in Fig. 7(a-e). The mean pressure field of Fig. 7(a) is characterised by leading and trailing edge shocks stemming from the upstream and downstream cavity edges. The leading edge shock stems at approximately the local Mach angle $\left(\arcsin 1.5^{-1}\right)$ to the inflow direction. The trailing edge shock is wider than the leading edge one, indicating the presence of a significant area of high pressure over the rear edge. This area extends to the top portion of the forward-facing rear wall, with a local maximum just upstream of the rear edge. This time-mean wall pressure trend ties with past experimental wall pressure measurement ${ }^{25}$ and with past numerical studies at the same inflow conditions. ${ }^{10}$ The most energetic POD mode, after the mean flow, is shown in Fig. 7(b). This POD basis of aerodynamic pressure identifies a longitudinal mode inside the enclosure, similar to the one that would result from a streamwise standing pressure wave. This mode features pressure anti-nodes at both ends of the cavity and a further pressure antinode is located in the middle of the opening. The pressure fluctuations are largest along the cavity shear layer, where the convecting pressure field of the vortices interferes with the upstream travelling pressure waves to generate the standing wave pressure pattern. The presence of three anti-nodes along the enclosure is consistent with a Rossiter mode number of two. Figures 7(c-e) describe progressively less energetic POD modes. These are not exclusively longitudinal instabilities as pressure anti-nodes are not aligned in the streamwise direction but show some flow normal displacement about the $y=0$ line. The propagation of
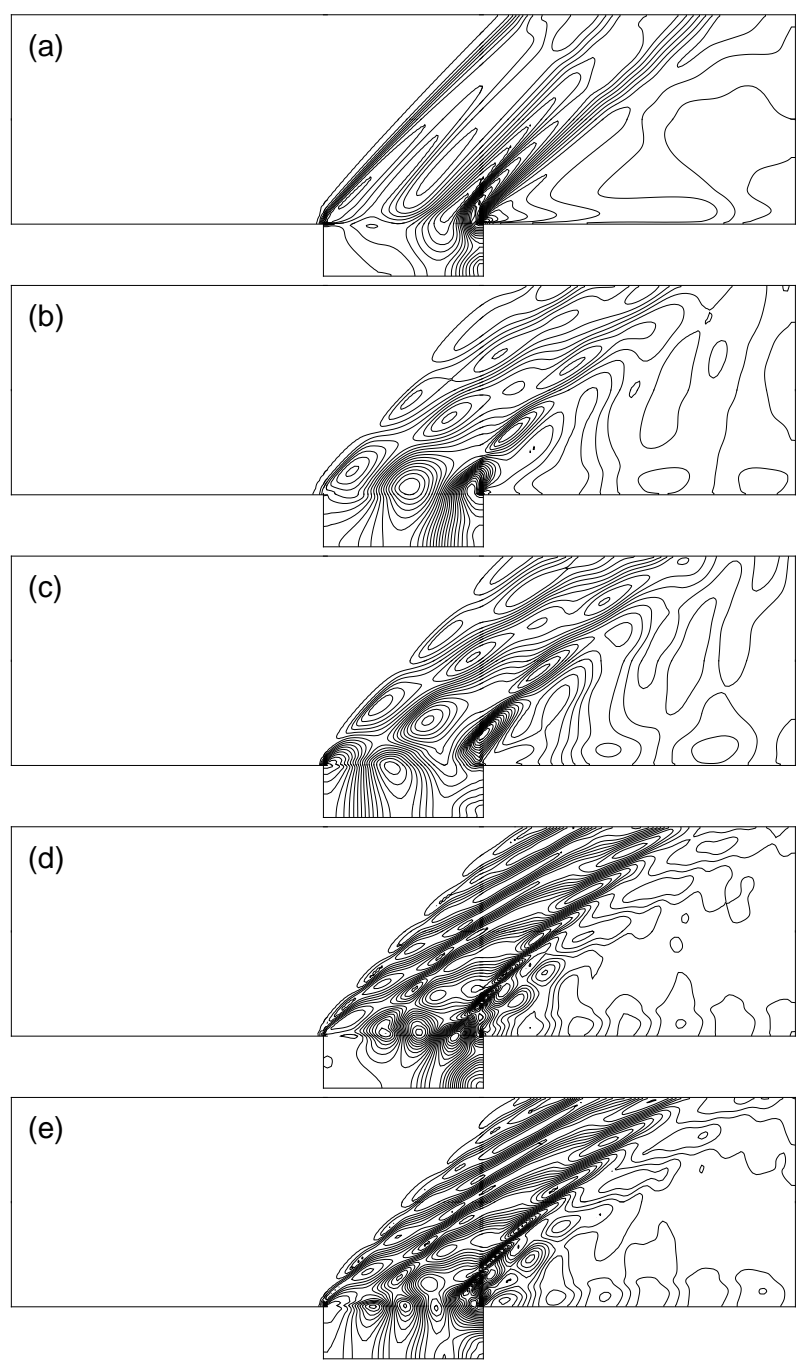

Fig. 7 POD modes. (a) mean flow, (b-e) first 4 most energetic modes.

pressure waves away from the enclosure to the far field are clearly shown above the enclosure in Figs. 7(b-e). The direction of propagation is similar to the direction of the upstream shock in Fig. 7(a).

A reconstruction of the pressure field has been performed to test this modal description of the flow. The reconstruction is obtained as described by Eq. 6 using the first 9 modes $(P=9)$. Four snapshots have been reconstructed, corresponding to the same phases of flow instability of Figs. 5(a-d). The reconstructed fields, shown in Figs. 8(a-d), are very similar to the benchmark snapshots of Figs. 5(a-d). All main features of the unsteady pressure field have been adequately reconstructed, including the low pressure areas along the shear layer that are associated to the downstream convection of vortices and the upstream propagation of pressure waves. A minor smearing of the wave fronts can be noticed from an accurate comparison between Figs. 5(a-d) and Figs. 8(a-d). This

6 of 9

American Institute of Aeronautics and Astronautics 

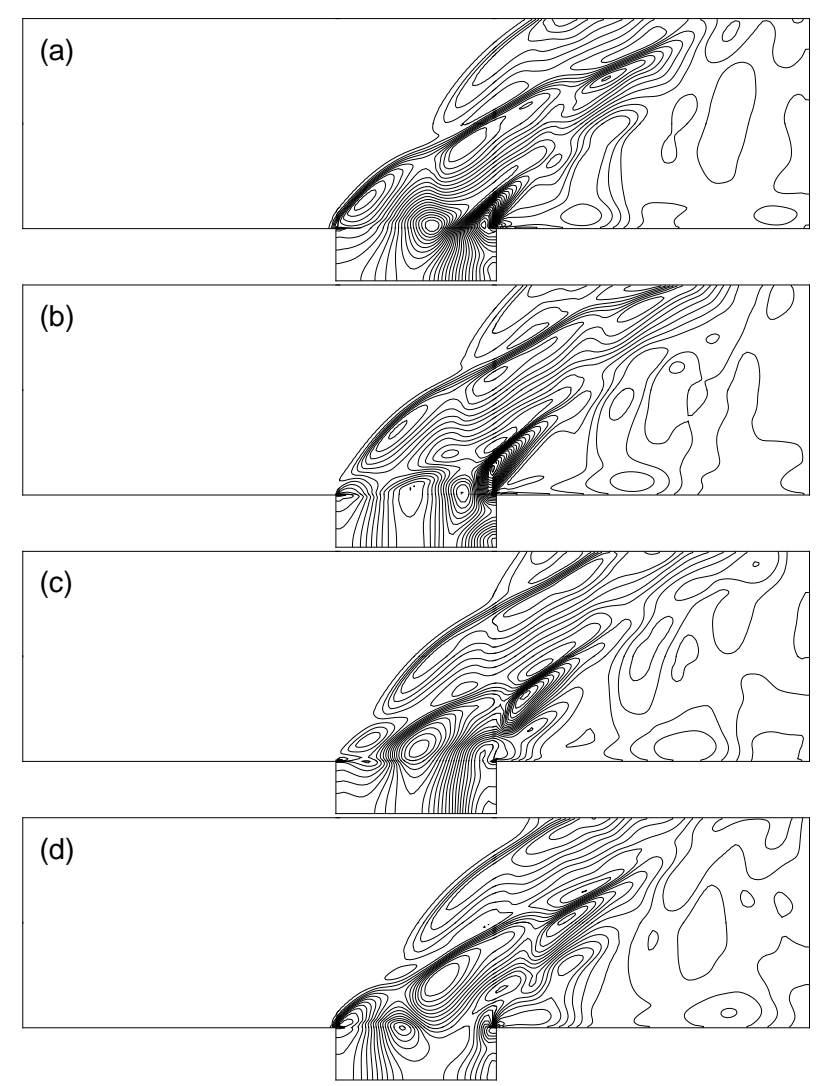

Fig. 8 Reconstructed cavity pressure contours over one dominant eigen-mode period $T$. (a) $t=0$, (b) $t=0.25 T$, (c) $t=0.5 T$, (d) $T=0.75 T . \Delta p=$ $0.003 \rho_{\infty} U_{\infty}^{2}$.

is due to the truncation of higher wavenumber modes in the reconstruction and does not alter the essential characters of the flow instability which is correctly preserved in the POD results.

Figure 9 shows the time history of four of the nine POD mode coefficients $\alpha_{i k}, k=1$ to 4 , that have been used in the reconstruction of the unsteady pressure snapshots of Figs. 8(a-d). The coefficients for the two leading modes have peak to peak fluctuation amplitudes well above those for modes 3 and 4 . This suggests that a reconstruction of the unsteady pressure field with only the mean flow and the first two modes may still give an adequate description of the pressure fluctuations in the enclosure, due to the tonal flow instability. In fact, from Fig. 6, about $92 \%$ of the model pressure fluctuation is due to the first two modes. This may lead to a further reduction in the complexity of the unsteady pressure predictor for flow control applications. The time traces of the POD mode coefficients are sinusoidal and conform to the expectations of a narrow-band frequency content expressed in the subsection 'Part 2: Discrete Fourier Series'.

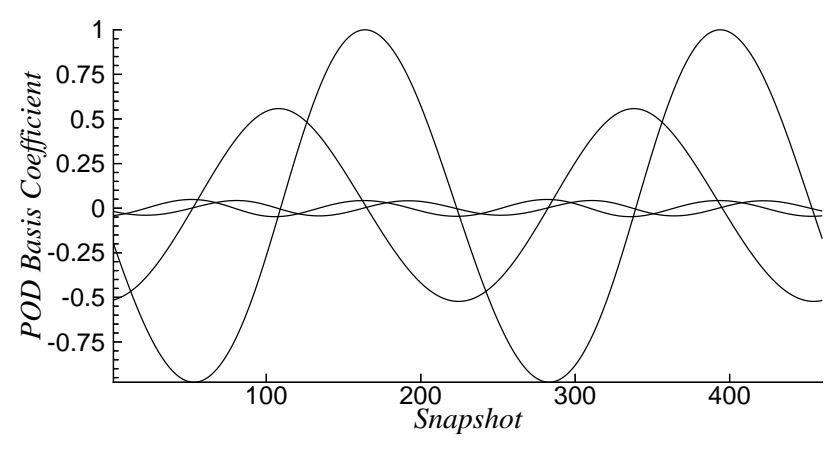

Fig. 9 POD $\alpha_{i k}$ coefficients for $k=1$ to 4 .

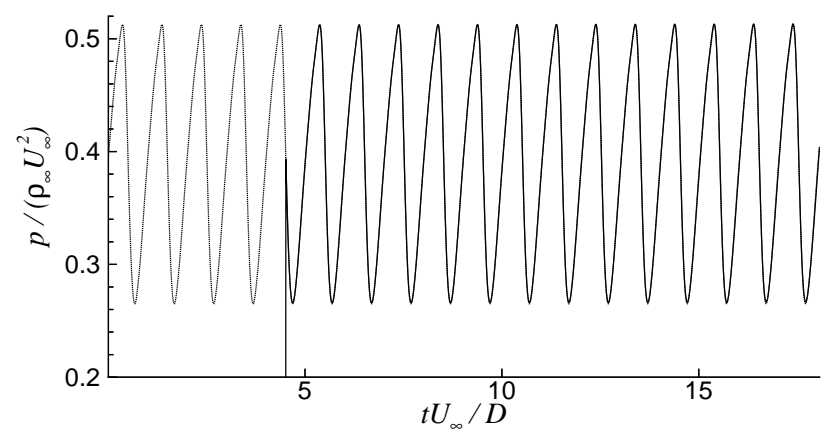

Fig. 10 Comparison of computed (dotted) and POD extrapolated (solid) pressure at the downstream edge. $2.5 T$ were used to generate the POD bases.

\section{POD pressure prediction}

As discussed in section 'Numerical Method', the explicit finite volume time-marching scheme has provided 18 periods, or 4147 snapshots, of stationary cavity flow data. A subset of the first 7 periods are used to construct the POD eigen-modes, as shown in Fig. 3. Specifically, this construction is based on a variable number of snapshots covering $0.5 n T, n$ being integer, ending at the end of the 7 th period. The POD pressure prediction begins at the beginning of the 8th period and continues to the end of the 18th period. A comparison between the POD wall pressure prediction and the CFD prediction is performed between period 8 and 18 and is presented in Figs. 10 and 11. The overall agreement between the two methods is good and the pressure traces are shown to overlap.

Figure 12 shows in details the comparison among the 18th period model pressure histories. Using 2.5 periods of CFD data to construct the POD bases gives a POD pressure fluctuation that overlaps with the CFD data. It appears that using POD bases from 3.0 periods introduces a small drift in phase in the POD extrapolation of the wall pressure in time. This difference has been further investigated by performing a quantitative error analysis on this data. This is referred to as Error Analysis in Fig. 3. An error indicator has been defined as the RMS difference between the POD and the CFD predictions, evaluated over the 


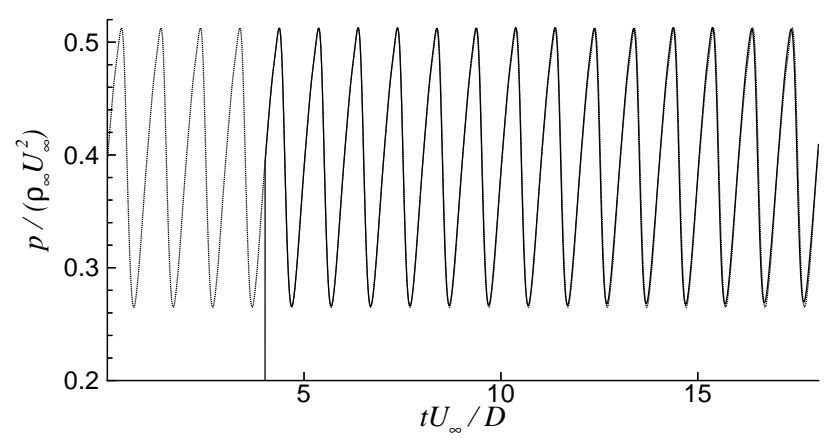

Fig. 11 Comparison of computed (dotted) and POD extrapolated (solid) pressure at the downstream edge. $3.0 T$ were used to generate the POD bases.

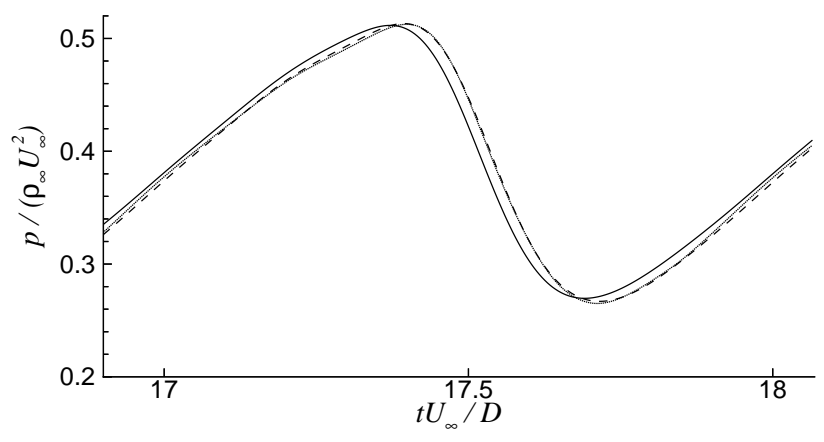

Fig. 12 Comparison of computed (dotted) and POD extrapolated pressure at the downstream edge. 2.5T (dashed line) and 3.0T (solid line) were used to generate the POD bases.

18th period. Specifically,

$$
\epsilon=\left\{\frac{1}{T} \sum_{t=17 T}^{18 T}\left[p_{\phi}(t)-p(t)\right]^{2} \Delta t\right\}^{0.5}
$$

where $p_{\phi}$ is the POD prediction, $p$ is the CFD reference prediction and $\Delta t=T / 230$. Figure 13 shows that the error associated to the $3.0 T$ POD predictions is about five times higher than the one associated to predictions that use $2.5 T$ to create the POD bases. While increasing the number of periods used to construct the POD bases generally decreases the RMS error, RMS error maxima occur when integer numbers are used while error minima occur when $(n+0.5) T$ are used.

\section{Conclusions}

Dynamic modelling of a supersonic turbulent cavity flow has been investigated by combining 'classical' computational fluid dynamics with a POD model reduction technique. The time dependent computational fluid dynamic model predicts self-sustained instabilities at the selected free stream conditions, in agreement with past experimental and numerical work. These instabilities show features that are typical of a limit cycle 'saturated' dynamic system, such

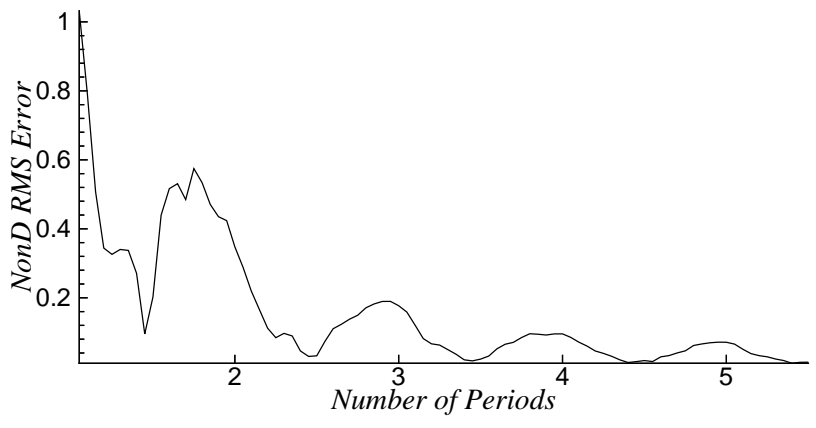

Fig. 13 Normalised RMS difference over the 18th period between the CFD and the POD predictions.

as a narrow-band frequency content and a significant phase coherence between successive cycles of flow oscillation. In addition, the proper orthogonal decomposition showed that the unsteady flow contains the equivalent of narrow-band wavenumbers in space, which tieup with the dominant narrow-band (frequency) modes to define, essentially, a low order dynamic flow instability.

The POD analysis was applied specifically to the unsteady pressure field. This exerts high aerodynamic loads on the cavity perimeter and is therefore most relevant to practical engineering applications. The unsteady pressure field at different phases in the instability cycle was reconstructed from a weighted addition of 9 POD bases. The results indicate that the reconstruction retained a high fidelity to the benchmark CFD predictions and most of the salient flow features that were adequately reproduced. The dominance of the second Rossiter mode gives scope to further reduce the number of POD bases in future work.

The flow pressure history was then extrapolated beyond the initial dataset by discrete Fourier transform. This method allowed to predict the pressure fluctuations without having to time step with a CFL limited $\Delta t$, unlike in the benchmark CFD method. The amplitude and phase coherence of the limit cycle type oscillation enabled a good prediction over 11 characteristic periods of the large-scale cavity flow instability. By eliminating the CFL constraint and limiting the predicted variables to the pressure field, a leaner numerical method was obtained, compared to the benchmark time marching explicit CFD scheme. Further computational benefits can be obtained by limiting the predictions to the known areas of largest aerodynamic load, such as the downstream edge, and by reducing the number of POD modes and of Fourier pairs used in the extrapolation. This will result in a reduction of computational resources and should open the route towards obtaining a real-time flow predictor for practical applications, such as for the active control aircraft store bay flow oscillations. 


\section{Acknowledgements}

The support of EPSRC grant GR/N23745 is acknowledged. This work was performed using the University of Leicester Mathematical Modelling Centre's supercomputer that was purchased through the EPSRC strategic equipment initiative.

\section{References}

${ }^{1}$ Arunajatesan, S., Shipman, J. D., and Sinha, N., "Hybrid RANS-LES simulation of cavity flow fields with control," Paper 2002-1130, AIAA, Jan. 2002.

${ }^{2}$ Stanek, M. J., Raman, G., Kibens, V., Ross, J. A., Odedra, D., and Peto, J. W., "Control of cavity resonance through very high frequency forcing," Paper 2000-1905, AIAA, Jan. 2000.

${ }^{3}$ Montegau, L., Kook, H., and Franchek, M. A., "Active control of flow-induced cavity resonance," Paper 98-2349, AIAA/CEAS, Jun. 1998.

${ }^{4}$ Lamp, A. M. and Chokani, N., "Control of cavity resonance using steady and oscillatory blowing," Paper 99-0999, AIAA, Jan. 1999.

${ }^{5}$ Williams, D. R., Rowley, C., Colonius, T., Murray, R., MacMartin, D., Fabris, D., and Albertson, J., "Model-based control of cavity oscillations - Part 1: Experiments," Paper 2002-0971, AIAA, Jan. 2002

${ }^{6}$ Grace, S. M., "An overview of computational aeroacoustic techniques applied to cavity noise prediction," Paper 2001-0510, AIAA, Jan. 2001.

${ }^{7}$ Shaw, L. L., "Weapons bay acoustic environment control," Paper 95-141, CEAS/AIAA, Jun. 1995.

${ }^{8}$ Rona, A., Aerodynamic and aeroacoustic estimations of oscillatory supersonic flows, Ph.D. thesis, University of Southampton, UK, Jun. 1997.

${ }^{9}$ Rona, A., Chen, X. X., Zhang, X., and Edwards, J. A., "Control of cavity flow oscillations through leading edge flow modifications," Paper 98-0672, AIAA, Jan. 1998.

${ }^{10}$ Zhang, X., Rona, A., and Edwards, J. A., "The effect of trailing edge geometry on cavity flow oscillation driven by a supersonic shear layer," The Aeronautical Journal, Vol. 102, No. 1013, May 1998, pp. 129-136.

${ }^{11}$ Zhang, X., Chen, X. X., Rona, A., and Edwards, J. A., "Attenuation of cavity flow oscillation through leading edge flow control," Journal of Sound and Vibration, Vol. 221, No. 1, 1999, pp. 23-47.

${ }^{12}$ Smith, B. R., Welterlen, T. D., Maines, B. H., Shaw, L. L., Stanek, M. J., and Grove, J. E., "Weapons bay acoustic suppression from rod spoilers," Paper 2002-0662, AIAA, Jan. 2002.

${ }^{13}$ Grove, J. E., Birkbeck, R. M., and Kreher, J. M., "Acoustic and separation characteristics with bay leading edge blowing," Paper 2000-1904, AIAA/CEAS, Jun. 2000.

${ }^{14}$ Rona, A. and Brooksbank, E. J., "Injection parameters for an effective passive control of cavity flow instability," Paper 2002-0119, AIAA, Jan. 2002

${ }^{15}$ Stanek, M. J., Raman, G., Kibens, V., Cain, A., and Lepicovsky, J., "Advanced actuator concepts for active aeroacoustic control," Paper 2000-1930, AIAA, Jan. 2000.

${ }^{16}$ Fabris, D. and Williams, D. R., "Experimental measurements of cavity and shear layer response to unsteady bleed forcing," Paper 99-0605, AIAA, Jan. 1999.

${ }^{17}$ Rowley, C. W., Williams, D. R., Colonius, T., Murray, R. M., MacMartin, D. G., and Fabris, D., "Model-based control of cavity oscillations Part II: System identification and analysis," Paper 2002-0972, AIAA, Jan. 2002.

${ }^{18}$ Zhang, X. and Edwards, J. A., "An investigation of supersonic oscillatory cavity flows driven by thick shear layers," The Aeronautical Journal, Vol. 94, No. 940, December 1990, pp. 355-364.
${ }^{19}$ Zhang, X., "Compressible cavity flow oscillation due to shear layer instabilities and pressure feedback," AIAA Journal, Vol. 33, No. 8, Aug. 1995, pp. 1404-1411.

${ }^{20}$ Zhang, X. and Edwards, J. A., "Analysis of unsteady cavity flow employing an adaptive meshing algorithm," Computers \& Fluids, Vol. 25, No. 4, May 1996, pp. 373-393.

${ }^{21}$ Freund, J. B. and Colonius, T., "POD analysis of sound generation by a turbulent jet," Paper 2002-0072, AIAA, Jan. 2002.

${ }^{22}$ Rowley, C. W., Colonius, T., and Murray, R. M., "POD based models of self-sustained oscillations in the flow past an open cavity," Paper 2000-1969, AIAA, Jan. 2000.

${ }^{23}$ Brooksbank, E. J. and Rona, A., "POD analysis and prediction of cavity flow instability," ICSV9 Paper 700, Ninth International Congress on Sound and Vibration, Orlando, Florida, USA, Jul. 2002.

${ }^{24}$ Charwat, A. F., Roos, J. N., Dewey, F. C. J., and Hitz, J, A., "An investigation of separated flow - Part I: The pressure field," J. of the Aerospace Sciences, Vol. 28, June 1961, pp. 457470 .

${ }^{25}$ Zhang, X., An experimental and computational investigation into shear layer driven single and multiple cavity flowfields, Ph.D. thesis, University of Cambridge, UK, 1988.

${ }^{26}$ Zhang, X., Rona, A., and Edwards, J. A., "An observation of pressure waves around a shallow cavity," J. Sound and Vibration, Vol. 214, No. 4, July 1998, pp. 771-778.

${ }^{27}$ Lumley, J. L., "The structure of inhomogeneous turbulent flows," Atm. Turb. and Radio Wave Prop., 1967, pp. 166-178.

${ }^{28}$ Rona, A. and Dieudonné, W., "Unsteady laminar and turbulent cavity flow models by second order upwind methods," Paper 99-0656, AIAA, Jan. 1999.

${ }^{29}$ Colonius, T., "An overview of simulation, modeling, and active control of flow/acoustic resonance in open cavities," $\mathrm{Pa}$ per 2001-0076, AIAA, Jan. 2001.

${ }^{30}$ Shieh, C. M. and Morris, P. J., "Comparison of twoand three-dimensional turbulent cavity flows," Paper 2001-0511, AIAA, Jan. 2001.

${ }^{31}$ Lilley, G. M., Zhang, X., and Rona, A., "Progress in computational aeroacoustics in predicting the noise radiated from turbulent flows," Int. J. Acous. and Vib., Vol. 2, No. 1, May 1997, pp. 2-9.

${ }^{32}$ Kok, J. C., "Resolving the dependence on free-stream values for the k-omega turbulence model," AIAA Journal, Vol. 38, No. 7, July 2000, pp. 1292-1295.

${ }^{33}$ Roe, P. L., "Approximate Riemann solvers, parameter vectors, and difference schemes," J. Comp. Physics, Vol. 43, 1981, pp. 357-372.

${ }^{34}$ Roe, P. L., "Some contributions to the modeling of discontinuous flows," Lectures in Applied Math. 22, Am. Math. Soc., 1985 .

${ }^{35} \mathrm{Hu}$, F. Q., Hussaini, M. Y., and Manthey, J., "Application of low dissipation and dispersion Runge-Kutta schemes to benchmark problems in computational aeroacoustics," ICASE/LaRC workshop on benchmark problems in computational aeroacoustics (CAA), edited by J. C. Hardin, J. R. Ristorcelli, and C. K. W. Tam, NASA Conference Publication 3300, May 1995, pp. 73-98.

${ }^{36}$ Rona, A. and Bennett, W. P., "Non-uniform total temperature in a turbulent vortex street," Paper 2001-2467, AIAA, Jun. 2001.

${ }^{37}$ Rossiter, J. E., "Wind tunnel experiments on the flow over rectangular cavities at subsonic and transonic speeds," Rep. \& Memo. 4328, Aero. Res. Council, Oct. 1964.

${ }^{38}$ Rona, A. and Dieudonné, W., "A flow-resonant model of transonic laminar open cavity instability," Paper 2000-1967, AIAA, Jan. 2000. 